\title{
Unveiling the AGN powering the "Composite" Seyfert/Star-forming galaxy NGC 7679: BeppoSAX and ASCA results
}

\author{
R. Della Ceca ${ }^{1}$, S. Pellegrini ${ }^{2}$, L. Bassani ${ }^{3}$, V. Beckmann ${ }^{4}$, M. Cappi ${ }^{3}$, G. G. C. Palumbo ${ }^{2,5}$, \\ G. Trinchieri ${ }^{1}$, and A. Wolter ${ }^{1}$ \\ 1 Osservatorio Astronomico di Brera, via Brera 28, 20121 Milan, Italy \\ 2 Università di Bologna, Dipartimento di Astronomia, via Ranzani 1, 40127 Bologna, Italy \\ 3 Istituto TeSRE/CNR Via Gobetti 101, 40129 Bologna, Italy \\ 4 INTEGRAL Science Data Centre, Chemin d'Ecogia 16, 1290 Geneva, Switzerland \\ 5 Agenzia Spaziale Italiana (ASI), Viale Liegi 26, 00198 Rome, Italy \\ Received 19 February 2001 / Accepted 5 June 2001
}

\begin{abstract}
We discuss BeppoSAX observations and archive ASCA data of NGC 7679, a nearby, nearly face-on SB0 galaxy in which starburst and AGN activities coexist. The X-ray observations reveal a bright $\left(L_{0.1-50} \mathrm{keV} \sim\right.$ $2.9 \times 10^{43} \mathrm{erg} \mathrm{s}^{-1}$ ) and variable source having a minimum observed doubling/halving time scale of $\sim 10-20 \mathrm{ksec}$. A simple power law with photon index of $\Gamma \sim 1.75$ and small absorption $\left(N_{\mathrm{H}}<4 \times 10^{20} \mathrm{~cm}^{-2}\right)$ can reproduce the NGC 7679 spectrum from 0.1 up to $50 \mathrm{keV}$. These X-ray properties are unambiguous signs of Seyfert 1 activity in the nucleus of NGC 7679. The starburst activity, revealed by the IR emission, optical spectroscopy and $\mathrm{H} \alpha$ imaging, and dominating in the optical and IR bands, is clearly overwhelmed by the AGN in the X-ray band. Although, at first glance, this is similar to what is observed in other starburst-AGN galaxies (e.g. NGC 6240, NGC 4945), most strikingly here and at odds with the above examples, the X-ray spectrum of NGC 7679 does not appear to be highly absorbed. The main peculiarity of objects like NGC 7679 is not the strength of their starburst but the apparent optical weakness of the Seyfert 1 nucleus when compared with its X-ray luminosity. To date NGC 7679 is one of the few Seyfert 1/Starburst composites for which the broad-band X-ray properties have been investigated in detail. The results presented here imply that optical and infrared spectroscopy could be highly inefficient in revealing the presence of an AGN in these kinds of objects, which instead is clearly revealed from X-ray spectroscopic and variability investigations.
\end{abstract}

Key words. galaxies: active - galaxies: individual: NGC 7679 - galaxies: Seyfert - galaxies: starburst - X-rays: galaxies

\section{Introduction}

The connection between starburst and AGN activity is one of the most discussed and debated topics of modern cosmology. This connection has been used to explain the overall properties and the physical nature of the Ultraluminous Infrared Galaxies (ULIRG, i.e. sources with bolometric luminosities in excess of $10^{12} L_{\odot}$, mostly emitted in the mid and far infrared), and recently (Fabian et al. 1998) as an elegant way to produce absorption in low luminosity $\left(L_{x} \sim 10^{43} \mathrm{ergs} \mathrm{s}^{-1}\right)$ AGNs. Therefore, understanding this phenomenon is clearly of high priority (see Veilleux 2000 for a recent review).

$\mathrm{X}$-ray spectroscopy is a very powerful tool to shed light on the starburst-AGN connection, mainly because X-rays are emitted closer to the primary emitting source than

Send offprint requests to: R. Della Ceca,

e-mail: rdc@brera.mi.astro.it optical emission lines, which represent reprocessed radiation from interstellar gas (e.g., Terashima et al. 2000). In addition, while the optical line diagnostic tools, based on emission line intensity ratios, are often compatible with both starburst and AGN activity (Terlevich et al. 1992), the X-ray properties of starbursts (i.e. mostly thermal emission) and AGNs (i.e. mostly non-thermal power-law emission) are quite different. In "pure" starburst galaxies, X-rays likely derive from stellar objects (type II supernovae, O stars, high and low mass X-ray binaries) as well as galactic winds and superwinds (see Heckman 2000 and references therein). X-ray observations of starburst galaxies (see e.g. Della Ceca et al. 1997; Dahlem et al. 1998; Ptak et al. 1999; Cappi et al. 1999; Pietsch et al. 2001; Griffiths et al. 2000) revealed very complex spectra, which required the presence of at least two or three thermal emission components with temperatures up to a few keV. If coming from "pure" AGN-like activity rather 
than from a starburst, the nuclear X-ray emission is expected to be stronger, more compact, variable, harder and described, to a first approximation, by a power law model with photon index $\Gamma \simeq 1.9 \pm 0.15$ (e.g., Nandra et al. 1997); iron-K fluorescence emission lines with a mean equivalent width $(E W)$ of $\sim 230 \mathrm{eV}$ are also often present (e.g., Nandra et al. 1997). If the nuclear emission intercepts absorbing material (e.g. a molecular torus), absorption effects (low energy cutoff, strong iron$\mathrm{K}$ fluorescence emission lines) are expected (see Bassani et al. 1999 and references therein). Starburst/Seyfert composites have been also found and studied in detail in the last years; it is now clear that heavy absorption $\left(N_{\mathrm{H}}>10^{23} \mathrm{~cm}^{-2}\right)$ in the nuclear region and a strong circumnuclear starburst are responsible for the broad-band properties of most of these objects (see Levenson et al. 2001; Iwasawa 1999 and references therein); their X-ray spectra are usually described by a combination of reflected/trasmitted AGN emission plus starburst thermal emission.

A case study to investigate the starburst-AGN connection is the barred, nearly face-on $\left(i \sim 30^{\circ}\right)$ SB0 galaxy NGC 7679 (also known as MKN 534 and Arp 216; $z=0.0177$, Kewley et al. 2001). Whether an active nucleus is present in NGC 7679, and to what extent starburst and AGN activities power the optical emission lines, or in general the total emission, is not clear yet (see Sect. 2). We present here results obtained from a BeppoSAX observation and archive ASCA data which help us clarify the nature of this galaxy.

\section{Why NGC 7679 ?}

NGC 7679 was originally selected by us for investigation of its starburst properties in the X-ray band. In fact, the $\mathrm{H} \alpha$ image reveals a luminous nuclear starformation complex resolved into bright clumps of emission and a bright circumnuclear starburst region that is about $18^{\prime \prime}(\sim 9.3 \mathrm{kpc})$ in diameter (Pogge \& Eskridge 1993). NGC 7679 also shows quite a strong infrared luminosity from IRAS data $\left(\log L_{\mathrm{FIR}} / L_{\odot}=11.10\right)$, a ratio $L_{\mathrm{FIR}} / L_{\mathrm{B}} \sim 1$, and infrared colors typical of a starburst galaxy (Kewley et al. 2001). Further support for the presence of a starburst in NGC 7679 are a) the existence of a bar; b) signs of tidal distortion due to the interaction with a faint companion $\sim 1^{\prime}$ east; and c) the physical association with the Seyfert 2 galaxy NGC 7682 (that lies $\sim 4.5$ east), as revealed by a stream of ionized gas connecting the two galaxies (Durret \& Warin 1990). These characteristics are expected to enhance the gas flow towards the galaxy center and are thought to trigger a starburst (see e.g. Combes 2000 for a review of this topic).

Optical spectroscopy by Kewley et al. (2001) reveals narrow optical emission lines (HII region-like) as well as a weak broad $\left(F W H M \sim 2000 \mathrm{~km} \mathrm{~s}^{-1}\right) \mathrm{H} \alpha$ component ${ }^{1}$.

\footnotetext{
1 We also have performed optical spectroscopic observations of NGC 7679 on September 141999 at the Mt. Orzale $152 \mathrm{~cm}$
}

Therefore, both the broad-line region of a central AGN and the narrow-line region dominated by the excitation of a starburst could have been detected in the optical spectrum $^{2}$.

Finally, NGC 7679 was previously imaged in X-rays with the Einstein Observatory IPC (890 s exposure, Fabbiano et al. 1992) and with the ROSAT PSPC during the ROSAT All Sky Survey (RASS) for a 381 s exposure (1RXS J232846.9+033042, www .xray.mpe.mpg.de/cgi-bin/rosat/rosat-survey). The Einstein IPC count rate $\left(2.7 \times 10^{-2}\right.$ counts s $\left.^{-1}\right)$, when converted using a $5 \mathrm{keV}$ thermal bremsstrahlung model filtered by Galactic absorption, gave a $(0.2-4) \mathrm{keV}$ flux of $1.1 \times 10^{-12} \mathrm{erg} \mathrm{cm}^{-2} \mathrm{~s}^{-1}$ and a luminosity of $1.5 \times 10^{42} \mathrm{erg} \mathrm{s}^{-1}$. The ROSAT PSPC $(0.1-2.4 \mathrm{keV})$ count rate is $(6.75 \pm 1.5) \times 10^{-2}$ counts $^{-1}$, corresponding to an observed flux (unabsorbed luminosity) of $9 \times 10^{-13} \mathrm{erg} \mathrm{cm}^{-2} \mathrm{~s}^{-1}\left(1.7 \times 10^{42} \mathrm{erg} \mathrm{s}^{-1}\right)$. No X-ray spectral information was available in literature prior to the results discussed here. Some of the main characteristics of NGC 7679 are reported in Table 1.

\section{BeppoSAX observations and data preparation}

NGC 7679 was observed by BeppoSAX on December 6, 1998 (the journal of the observation and source count rates are given in Table 2). In this paper we use data from three Narrow Field Instruments: the Low Energy Concentrator Spectrometer (LECS, Parmar et al. 1997), the Medium Energy Concentrator Spectrometer (MECS, Boella et al. 1997), and the Phoswich Detector System (PDS, Frontera et al. 1997). HPGSPC data (Manzo et al. 1997) are not considered since the source is too faint to be detected.

The cleaned and linearized data produced by the BeppoSAX Science Data Center (http:// www.asdc.asi.it/bepposax/) have been analyzed using standard software (XSELECT v1.4, FTOOLS $\mathrm{v} 4.2$ and XSPEC v10.0). The PDS data reduction was performed using XAS (V2.0, Chiappetti \& Dal Fiume 1997) and SAXDAS (v1.3.0) software packages and yielded consistent results.

telescope of the Osservatorio Astronomico di Bologna. The observed optical spectral properties (line features, FWHM and ratios) support the classification of NGC 7679 as a starburst galaxy (cf. Veilleux \& Osterbrock 1987; Ho et al. 1997), in good agreement with the results obtained independently by Kewley et al. (2001). We do not detect the broad $\mathrm{H} \alpha$ component at the bottom of the strong and narrow $\mathrm{H} \alpha$ line, but the $\mathrm{S} / \mathrm{N}$ ratio of our spectra is probably not adequate for this purpose.

2 A caveat, however, is in order. The $F W H M\left(\sim 2000 \mathrm{~km} \mathrm{~s}^{-1}\right)$ and luminosity $\left(\sim 8 \times 10^{40} \mathrm{erg} \mathrm{s}^{-1}\right.$, Keweley, private communication) of the broad $\mathrm{H} \alpha$ component detected in NGC 7679 could in principle be produced by a supernova explosion. A clear example is the type II Supernova 1997ab found in the dwarf galaxy HS 0948+2018 (Hagen et al. 1997); this type II supernova has a broad $\left(F W H M \sim 2500 \mathrm{~km} \mathrm{~s}^{-1}\right) \mathrm{H} \alpha$ component with a luminosity that is more than an order of magnitude higher than that of NGC 7679 . 
Table 1. General characteristics of NGC 7679.

\begin{tabular}{llllllllll}
\hline Type $^{a}$ & $\begin{array}{l}\text { RA } \\
(\mathrm{J} 2000)\end{array}$ & $\begin{array}{l}\text { Dec } \\
(\mathrm{J} 2000)\end{array}$ & $\begin{array}{l}d^{b} \\
(\mathrm{Mpc})\end{array}$ & $\begin{array}{l}\text { Optical }^{b} \\
\text { classification }\end{array}$ & $\begin{array}{l}B_{\mathrm{T}}^{0}{ }^{a} \\
(\mathrm{mag})\end{array}$ & $\begin{array}{l}\log L_{B}{ }^{a} \\
\left(L_{\odot}\right)\end{array}$ & $\begin{array}{l}\log L_{\mathrm{FIR}}{ }^{b} \\
\left(L_{\odot}\right)\end{array}$ & $\begin{array}{l}\operatorname{Size}^{a} \\
(\operatorname{arcmin})\end{array}$ & $\begin{array}{l}N_{\mathrm{H}, \mathrm{Gal}}{ }^{c} \\
\left(\mathrm{~cm}{ }^{-2}\right)\end{array}$ \\
\hline $\mathrm{SB} 0$ & $23^{\mathrm{h}} 28^{\mathrm{m}} 46^{\mathrm{s}} .8$ & $03^{\circ} 30^{\prime} 41^{\prime \prime}$ & 107 & SB+AGN & 12.89 & 11.09 & 11.10 & $1.3 \times 0.9$ & $4.54 \times 10^{20}$ \\
\hline
\end{tabular}

${ }^{a}$ From de Vaucouleurs et al. (1991). $B_{\mathrm{T}}^{0}$ is the total $B$ magnitude, corrected for Galactic and internal extinction. The total $B$ band luminosity, $L_{B}$, has been derived using the indicated distance and $B_{\mathrm{T}}^{0}$.

${ }^{b}$ From Kewley et al. (2001). Distance based on $H_{0}=50 \mathrm{~km} \mathrm{~s}^{-1} \mathrm{Mpc}^{-1}, q_{0}=0$ and $z=0.0177$; at this distance $1^{\prime}=31.13 \mathrm{kpc}$. The infrared luminosity has been adapted for $H_{0}=50 \mathrm{~km} \mathrm{~s}^{-1} \mathrm{Mpc}^{-1}$.

${ }^{c}$ Galactic neutral hydrogen column density along the line of sight from the Leiden/Dwingeloo Survey (Hartman \& Burton 1997).

NGC 7679 is detected with a high signal-to-noise ratio $(S / N)$ in the LECS and MECS instruments (see Table 2) but is not spatially resolved; the center of the X-ray emission was found to be coincident, within the positional accuracy allowed by BeppoSAX data, with its optical nucleus. To maximize the statistics and the $S / N$, the MECS and LECS source counts have been extracted from a circle of $4^{\prime}$ radius around the centroid of the X-ray emission. Background counts were extracted from high Galactic latitude "blank" fields (provided by the BeppoSAX Science Data Center) using an extraction region which corresponds in size and detector position to that used for the source.

A very small elongation (at a few percent of the peak level) appears in the MECS image at the position of the Seyfert 2 galaxy NGC 7682, which lies 4.5 east of NGC 7679 (see Sect. 2). NGC 7682 is not detected in the ROSAT All Sky Survey; from the original RASS data we can evaluate a flux upper limit $(0.1-2.4 \mathrm{keV})$ which is at least a factor of 10 fainter than that of NGC 7679. To investigate if this source could contaminate the spectral results on NGC 7679 we have excluded the MECS counts deriving from a circle of $2^{\prime}$ radius centered on the position of NGC 7682, obtaining essentially the same results as those reported in Sect. 5 .

Another X-ray source is detected with $S / N \sim 10$ in the MECS image at about $19^{\prime}$ to the southwest of NGC 7679 (RA $=23^{\mathrm{h}} 27^{\mathrm{m}} 35 \mathrm{~s} .8$, Dec $=$ $\left.03^{\circ} 22^{\prime} 50^{\prime \prime}\right)$. A RASS object (1RXS J232735.2+032334, www . xray. mpe.mpg.de/cgi-bin/rosat/rosat-survey) is positionally consistent with this source; the RASS count rate is $0.03 \pm 0.01$, corresponding to an observed flux $(0.07-2.4 \mathrm{keV})$ of $\sim 4 \times 10^{-13} \mathrm{erg} \mathrm{cm}^{-2} \mathrm{~s}^{-1}$. There are no cataloged NED or SIMBAD objects inside a circle of $2^{\prime}$ radius from the RASS position.

Finally, there is a $6 \sigma$ detection in the PDS, also after conservative subtraction of the systematics residuals (currently evaluated at $\sim 0.02$ counts $\mathrm{s}^{-1}$ in the $13-200 \mathrm{keV}$ band, Guainazzi \& Matteuzzi 1997). There are no cataloged and bright $(2-10 \mathrm{keV}) \mathrm{X}$-ray sources, other than NGC 7679, in the PDS field of view.

\section{X-ray variability}

The LECS and MECS light curves extending over the whole observing period are shown in Figs. 1 (LECS) and 2 (MECS). A flux increase of roughly a factor 2.4 is present between $\sim 9 \times 10^{4}$ and $\sim 1.3 \times 10^{5}$ s from the start of the observation in both LECS and MECS data. The flux decreases afterwards, until the end of the observation, reducing itself by a factor of three. No equivalent variations in the background are found. This trend is present in all the energy bands considered (i.e., $0.1-2$ and $2-4 \mathrm{keV}$ for the LECS, and 1.65-4 and 4-10 keV for the MECS). The flux variability seems to be not associated with spectral variations (see ratios in Figs. 1 and 2) and, therefore, it has to be attributed to an overall continuum variation; this is confirmed by the spectral analysis reported in Sect. 5.2. From the flux change rate of the light curves we estimate a minimum doubling/halving time in the range 10-20 ksec. Variability on smaller timescales cannot be investigated due to the limited statistics available.

\section{X-ray spectral analysis}

Spectral channels corresponding to energies $0.12-4 \mathrm{keV}$ and 1.65-10 keV have been used respectively for the analysis of the LECS and MECS data (as suggested by the BeppoSAX Cookbook, Fiore et al. 1999). Spectral channels corresponding to energies $15-50 \mathrm{keV}$ have been used for the PDS data. LECS and MECS source counts have been rebinned to have a $S / N>5$ in each energy bin. Standard response matrices (September 1997 for MECS and January 2000 for LECS) and standard cross-constants normalizations for the instruments (see Fiore et al. 1999) have been used in the fitting procedure. All the models discussed in this paper have been filtered by the Galactic absorption column density $\left(N_{\mathrm{H}, \mathrm{Gal}}=4.54 \times 10^{20} \mathrm{~cm}^{-2}\right.$, see Table 1). Quoted errors on the fit parameters give the $90 \%$ confidence intervals for one interesting parameter $\left(\Delta \chi^{2}=2.71\right)$. Finally, thermal emission is modeled with the MEKAL plasma emission code. 
Table 2. BeppoSAX observation log.

\begin{tabular}{|c|c|c|c|c|c|c|c|}
\hline \multirow{2}{*}{$\begin{array}{l}\text { Sequence } \\
\text { Number }\end{array}$} & \multirow[t]{2}{*}{ Date } & \multicolumn{3}{|c|}{ Exposure time (ks) } & \multicolumn{3}{|c|}{ Count Rate $^{a}$ (counts s $^{-1}$ ) } \\
\hline & & LECS & MECS & PDS & $\begin{array}{l}\text { LECS } \\
0.12-4 \mathrm{keV}\end{array}$ & $\begin{array}{l}\text { MECS } \\
1.65-10 \mathrm{keV}\end{array}$ & $\begin{array}{l}\text { PDS } \\
15-50 \mathrm{keV}\end{array}$ \\
\hline 40631001 & 1998 Dec. 6-9 & 39.8 & 90.9 & 45.3 & $0.041 \pm 0.001$ & $0.073 \pm 0.001$ & $0.119 \pm 0.017$ \\
\hline
\end{tabular}

${ }^{a}$ Background subtracted source count rates, with $1 \sigma$ photon counting statistics errors. Extraction radii for LECS and MECS are $4^{\prime}$. The net counts from NGC 7679 represent about $92 \%$ of the total gross counts in the LECS or MECS source region. The PDS counts correspond to a $6 \sigma$ detection.

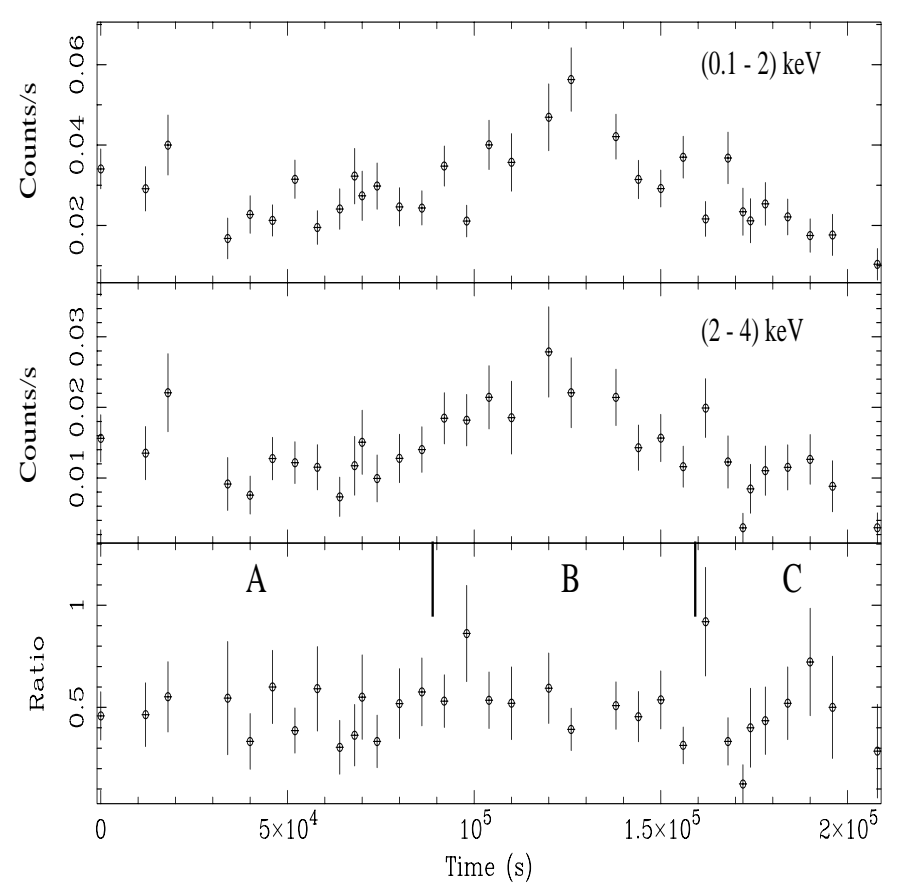

Fig. 1. The BeppoSAX LECS light curves and ratios of NGC 7679 in the $0.1-2$ and $2-4 \mathrm{keV}$ energy bands. Time bins of $2000 \mathrm{~s}$ have been used. The time intervals A, B, and C are those considered to investigate the spectral properties of NGC 7679 as a function of the source flux (see Sect. 5.1).

\subsection{Results}

In the fitting process we have used only LECS and MECS data, since the PDS data could be contaminated by the X-ray source located $\sim 19^{\prime}$ to the south-west of NGC 7679 (see Sect. 3 and below). However, the PDS data have been used to investigate the behavior of the NGC 7679 spectrum in the energy range $10-50 \mathrm{keV}$ and to check for consistency with the LECS/MECS results.

The simplest interpretation of the overall LECS and MECS data is a single power law model with photon index $\Gamma \sim 1.75$ and small intrinsic absorption, $N_{\mathrm{H}}<$ $4 \times 10^{20} \mathrm{~cm}^{-2}$ (see Table 3). No emission lines or absorption edges of statistical significance are found superimposed on the power law continuum; the $90 \%$ upper limits on the $\mathrm{Fe} \mathrm{K}$ lines equivalent width at 6.4 and $6.7 \mathrm{keV}$ are $180 \mathrm{eV}$ and $170 \mathrm{eV}$, respectively. From a statistical point of view $\left(\chi_{\nu}^{2}=0.93\right)$, no further spectral complexity is required.

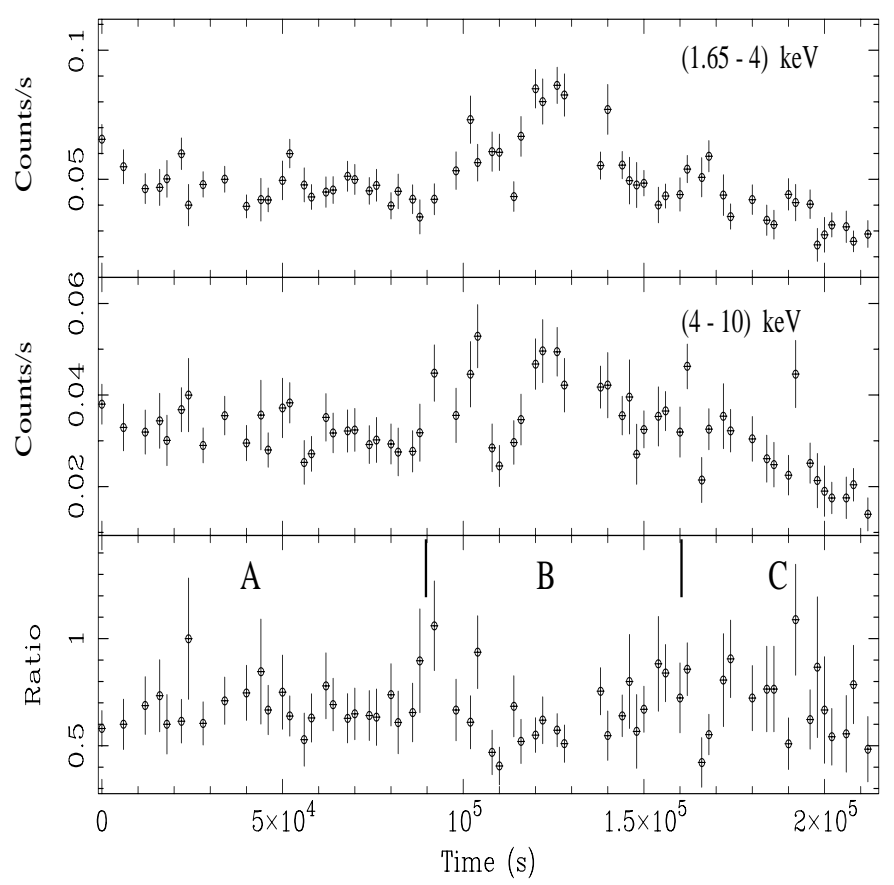

Fig. 2. The BeppoSAX MECS light curves and ratios of NGC 7679 in the $1.65-4$ and $4-10 \mathrm{keV}$ energy bands. Time bins of $2000 \mathrm{~s}$ have been used. The time intervals A, B, and C are those considered to investigate the spectral properties of NGC 7679 as a function of the source flux (see Sect. 5.1).

The observed spectra and the residuals of the LECS, MECS and PDS data set compared with the best fit power-law model are reported in Fig. 3. In the PDS energy range we have also included in the model the contribution expected from the X-ray source detected in the MECS field of view (see Sect. 3), which we estimate to be $\sim 20 \%^{3}$. The very good agreement between the PDS data and the extrapolation of the power-law model, which

${ }^{3}$ The MECS spectrum of this source is described by a power law model with photon index of $1.5_{-0.4}^{+0.5}$. The net counts are $245 \pm 23$, corresponding to a $2-10 \mathrm{keV}$ observed flux of $\sim 8 \times 10^{-13} \mathrm{erg} \mathrm{cm}^{-2} \mathrm{~s}^{-1}$. The RASS flux of this object (see Sect. 3) is consistent with that estimated using the MECS, therefore no sign of variability is present on long timescales. The PDS regime has been modeled with the same power law which describes the MECS data; extrapolated to the $10-50 \mathrm{keV}$ range the expected flux is $\sim 1.7 \times 10^{-12} \mathrm{erg} \mathrm{cm}^{-2} \mathrm{~s}^{-1}$, i.e. about $20 \%$ that from NGC 7679 itself. 


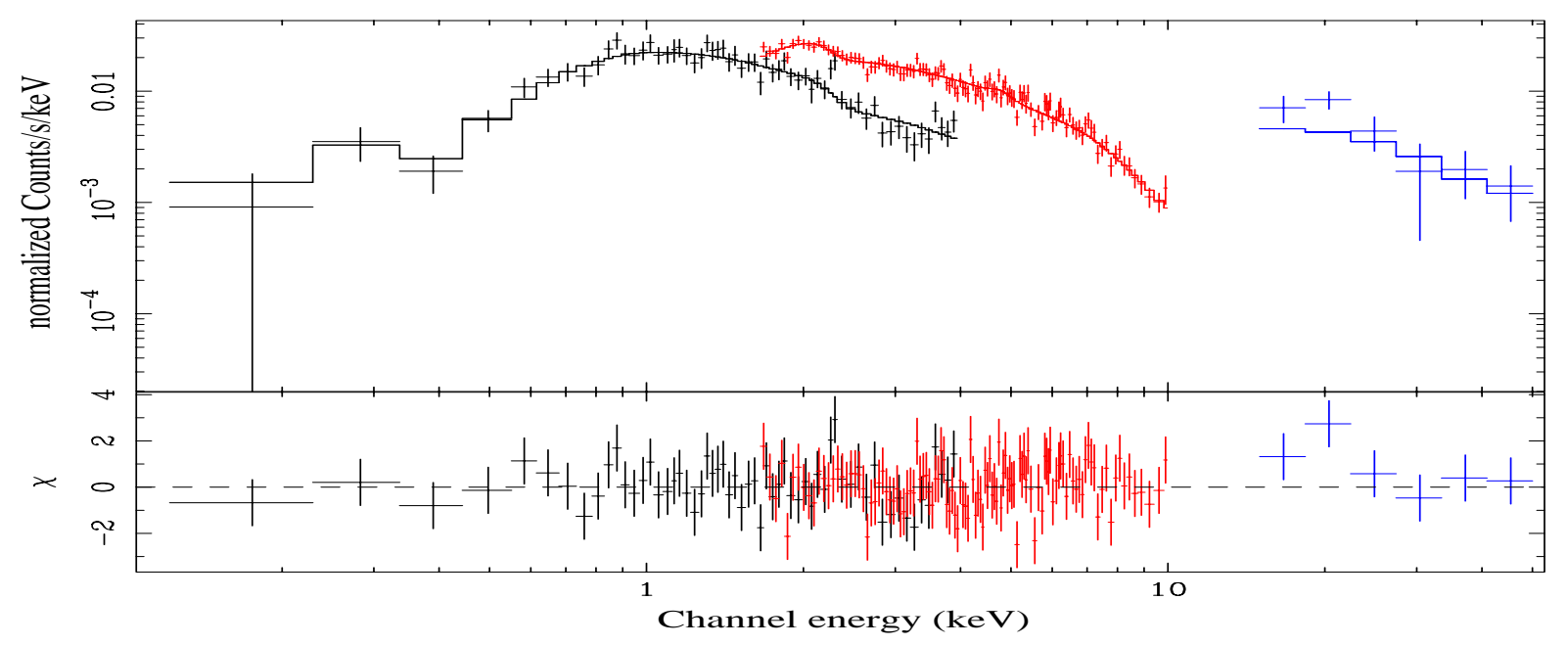

Fig. 3. The LECS, MECS and PDS folded spectra of NGC 7679 fitted with a power law (Table 3). The residuals of the fit are also shown.

describes the X-ray spectrum of NGC 7679 in the LECS and MECS energy range, implies that this simple model can reproduce the NGC 7679 spectrum from 0.1 up to $50 \mathrm{keV}$. The observed fluxes and intrinsic luminosities of the best fit models are given in Table 3 . The observed flux is $\sim 6$ times higher than that estimated from the Einstein IPC observation (0.2-4.0 keV energy range) and about a factor of 4 times higher than that measured from the PSPC during the ROSAT All Sky Survey (0.1-2.4 keV energy range).

We have also tried a model composed of a soft thermal component plus an absorbed power law component. Besides having been suggested as "canonical" model for a sample of low luminosity AGNs, LINERs and starburst galaxies observed with $A S C A$ (Ptak et al. 1999) such a model also describes the case of a starburst dominating the soft emission and a (heavily obscured) AGN dominating the hard one. Leaving the abundance as a free parameter, its best fit value is very low $(A \sim 0.01)$ and is probably unfeasible for a starburst. Fixing the abundance to the solar ones, the best fit values are $k T \sim 1.2 \mathrm{keV}$ and $\Gamma=1.72_{-0.12}^{+0.07}$, again with small intrinsic absorption $\left(N_{\mathrm{H}}<4.5 \times 10^{20} \mathrm{~cm}^{-2}\right)$, giving unabsorbed luminosities $(0.1-10 \mathrm{keV})$ of the thermal and power law components of $3.8 \times 10^{41} \mathrm{ergs} \mathrm{s}^{-1}$ and $1.6 \times 10^{43} \mathrm{ergs} \mathrm{s}^{-1}$, respectively. Such a soft thermal component could be accommodated within the data uncertainties but it is not statistically required by the $\mathrm{F}$ test $\left(\Delta \chi^{2}=1\right.$ for 2 additional parameters).

Finally, in order to study in detail the spectral properties of NGC 7679 as the flux level changes, we repeated the spectral analysis using the LECS and MECS counts ${ }^{4}$ from the three different time intervals shown in Figs. 1 and 2: A (quiescent status), B (flux increase) and C (flux decrease).

\footnotetext{
${ }^{4}$ Due to the limited statistics available in the PDS, it was not possible to split the observation in 3 segments. The PDS data will not be considered here.
}

Table 3. Results of the spectral analysis. Power law model.

\begin{tabular}{ll}
\hline \hline BeppoSAX LECS and MECS data \\
$N_{\mathrm{H}}\left(10^{20} \mathrm{~cm}^{-2}\right)$ & $2.2_{-1.4}^{+1.8}$ \\
$\Gamma$ & $1.75_{-0.05}^{+0.05}$ \\
$\chi^{2} / \nu / \chi_{\nu}^{2}$ & $160 / 172 / 0.93$ \\
Flux $^{a}\left(10^{-12} \mathrm{erg} \mathrm{cm}^{-2} \mathrm{~s}^{-1}\right)$ & $3.3,6.0,9.0$ \\
$L_{x}{ }^{a}\left(10^{42} \mathrm{erg} \mathrm{s}^{-1}\right)$ & $8.6,8.1,12.2$ \\
\hline \hline ASCA SIS and GIS data & \\
$N_{\mathrm{H}}\left(10^{20} \mathrm{~cm}^{-2}\right)$ & $<7.6$ \\
$\Gamma$ & $1.72_{-0.06}^{+0.06}$ \\
$\chi^{2} / \nu / \chi_{\nu}^{2}$ & $342 / 312 / 1.1$ \\
Flux $^{a}\left(10^{-12} \mathrm{erg} \mathrm{cm}^{-2} \mathrm{~s}^{-1}\right)$ & $2.8,5.2,8.3$ \\
$L_{x}{ }^{a}\left(10^{42} \mathrm{erg} \mathrm{s}^{-1}\right)$ & $7.0,7.1,11.2$ \\
\hline \hline
\end{tabular}

$N_{\mathrm{H}}$ is the column density of neutral hydrogen in addition to $N_{\mathrm{H}, \text { Gal }}$ given in Table 1. The LECS/MECS and the SIS/GIS normalizations ( 0.74 and 0.77 , respectively) are consistent with the known differences in the absolute calibration of the instruments.

${ }^{a}$ Observed fluxes and unabsorbed luminosities are given in the $0.1-2,2-10$, and $10-50 \mathrm{keV}$ bands. Fluxes and luminosities are relative to the MECS normalization (BeppoSAX data) or to the GIS normalization (ASCA data).

Each spectrum is well fitted by a single power law model with spectral parameters statistically consistent with those of the total spectrum (see Table 3). This confirms our expectations based on the constancy of the ratios of the count rates in various energy bands (see Sect. 4). No thermal components are required. There is some marginal evidence for the presence of line-like residuals around $7.1 \mathrm{keV}$ when NGC 7679 is brighter (time interval B), and around $6.2 \mathrm{keV}$ when NGC 7679 is weaker (time interval C). 


\section{ASCA observations}

NGC 7679 was observed by the ASCA satellite (Tanaka et al. 1994) on July 1, 1998. The data were retrieved from the ASCA archive and were cleaned by applying the standard selection criteria described in the TARTARUS database (see http: //tartarus.gsfc.nasa.gov) and using the same software packages used for the BeppoSAX data. The journal of the observation and source count rates are given in Table 4.

\subsection{Data preparation}

Total counts (source + background) were extracted from a circular region of $4^{\prime}$ radius for SIS0 and SIS1 and a $6^{\prime}$ radius for GIS2 and GIS3. For the SIS, background counts were taken from the remaining area of the chip, while for the GIS, background counts were taken from source-free circular regions close to NGC 7679. We exclude from the spectral analysis all SIS data with $E<1.0 \mathrm{keV}$ and all the GIS data with $E<0.6 \mathrm{keV}$ (see http://heasarc.gsfc.nasa.gov/docs/asca/watchout. html). No other X-ray sources are detected in the SIS or GIS field of view (note that the X-ray source detected in the MECS image, at about $19^{\prime}$ to the south-west of NGC 7679, is outside the ASCA GIS or SIS field of view).

For the GIS data we use the detector Redistribution Matrix Files (RMF) gis2v4_0.rmf and gis3v4_0.rmf, respectively; for the SIS data the RMF files were obtained using the FTOOLS task SISRMG. The Ancillary Response Files (ARF) for both SIS and GIS were created with the FTOOLS task ASCAARF at the location of NGC 7679 in the detectors.

In order to improve on the statistics we have produced a combined SIS spectrum (SIS01 hereafter) and a combined GIS spectrum (GIS23 hereafter) along with the relative background files and calibrations (see http:// heasarc.gsfc.nasa.gov/docs/asca/abc/abc. html). For the spectral analysis, source counts have been rebinned to have a $S / N>5$ in each energy bin.

\subsection{ASCA results}

The ASCA SIS and GIS light curves show a behavior very similar to those obtained using the BeppoSAX instruments (see Figs. 1 and 2), i.e. a variation of the count rates of a factor of 2-3 over the total observation, and a similar minimum doubling/halving time.

The ASCA (SIS01, GIS23) spectrum of NGC 7679 over the whole observing period is well described by a power law model with $\Gamma \sim 1.72$ and small intrinsic absorption, $N_{\mathrm{H}}<7.6 \times 10^{20} \mathrm{~cm}^{-2}$ (see Table 3 and Fig. 4 ). More complex models are not required statistically. As for the BeppoSAX data, no spectral variations were found as a function of the source count rate, as expected from the constancy (within the errors) of the ratios of the count rates in various energy bands (e.g., $0.6-2 \mathrm{keV}$ vs. 2-10 keV). There is some evidence for an FeK line in the
ASCA spectrum. Over the whole observing period, this line is located around $6.6 \mathrm{keV}$ (rest frame); in agreement with the BeppoSAX results the line best-fit energy also appears to be higher $(\sim 6.9 \mathrm{keV})$ when the source is brighter, and lower $(\sim 6.3 \mathrm{keV})$ when the source is weaker. However, the present statistics, either from BeppoSAX or ASCA, are too poor to be conclusive and require further observations by, e.g., XMM or Chandra. We note here only that such variability of the properties of the Iron lines has been found to be very common in a sample of Seyfert 1 galaxies investigated with ASCA (Weaver et al. 2001).

\section{Discussion}

\subsection{Starburst or AGN?}

All the X-ray $(0.1-50 \mathrm{keV})$ properties presented here (Sect. 4: variability; Sects. 5 and 6: spectra) provide strong evidence in favor of the existence of an AGN in NGC 7679. Indeed, as summarized below, the presence of an AGN is overwhelming in X-rays with respect to that of a starburst.

The minimum detectable doubling/halving variability time, $t_{\mathrm{var}} \sim 15 \mathrm{ksec}$, implies (using light crossing arguments) an emitting region with a size $\leq \sim 4.5 \times 10^{14} \mathrm{~cm}$ $\left(1.5 \times 10^{-4} \mathrm{pc}\right)$, much smaller than the starburst size $(\sim 10 \mathrm{Kpc})$. Furthermore, the maximum observed variation, $\Delta L_{2-10 \mathrm{keV}} \simeq 5 \times 10^{42} \mathrm{erg} \mathrm{s}^{-1}$, is very high for being produced inside a starburst nucleus.

A simple power-law spectral model $(\Gamma \sim 1.75)$ with small intrinsic absorption $\left(N_{\mathrm{H}}<4 \times 10^{20} \mathrm{~cm}^{-2}\right)$ provides a very good description of the spectral properties of NGC 7679 from 0.1 up to $50 \mathrm{keV}$. This spectral shape along with the variability properties closely resembles that typically observed in Seyfert 1 galaxies (Nandra et al. 1997).

Finally, the total X-ray luminosity of NGC 7679 $\left(L_{0.1-10 \mathrm{keV}} \sim 1.7 \times 10^{43} \mathrm{erg} \mathrm{s}^{-1}\right)$ also places it closer to AGNs than to starbursts. The most X-ray luminous star-forming galaxy yet detected, NGC 3256 (Moran et al. 1999 ), has $L_{0.1-10 \mathrm{keV}} \simeq 2.2 \times 10^{42} \mathrm{erg} \mathrm{s}^{-1}$, which is $\sim 8$ times weaker than that of NGC 7679. Note instead that the infrared luminosity of NGC $3256\left(L_{\mathrm{FIR}} \simeq 6 \times 10^{11} L_{\odot}\right)$ is $\sim 5$ times higher than that of NGC 7679 , and so the starburst is likely to be more powerful in NGC 3256 .

What about the possible starburst contribution to the X-ray emission? In Sect. 5.1 we have shown that a soft thermal component can be accommodated within the data uncertainties; its $(0.1-10 \mathrm{keV})$ luminosity $(\simeq 3.8 \times$ $\left.10^{41} \mathrm{erg} \mathrm{s}^{-1}\right)$ is $\sim 5$ times lower than the total thermal emission in NGC 3256, in better accordance with the lower infrared luminosity of NGC 7679.

\subsection{Which kind of AGN?}

Given that the bulk of the X-ray emission is powered by an AGN, in this section we try to specify which kind of AGN better accounts for the observed X-ray properties of NGC 7679. 
Table 4. ASCA observation log.

\begin{tabular}{|c|c|c|c|c|c|}
\hline \multirow{3}{*}{$\begin{array}{l}\text { Sequence } \\
\text { Number }\end{array}$} & \multirow[t]{3}{*}{ Date } & \multicolumn{2}{|c|}{ Exposure time (ks) } & \multicolumn{2}{|c|}{ Count Rate $^{a}$ (counts s ${ }^{-1}$ ) } \\
\hline & & SIS01 & GIS23 & SIS01 & GIS23 \\
\hline & & & & $1-10 \mathrm{keV}$ & $0.6-10 \mathrm{keV}$ \\
\hline 66019000 & 1998 July 1 & 88.8 & 98.3 & $0.088 \pm 0.001$ & $0.079 \pm 0.001$ \\
\hline
\end{tabular}

${ }^{a}$ Background subtracted source count rates, with $1 \sigma$ photon counting statistics errors. Extraction radii for SIS and GIS are $4^{\prime}$ and $6^{\prime}$, respectively. The net counts from NGC 7679 represent about $81 \%$ of the total gross counts in the GIS or SIS source region.

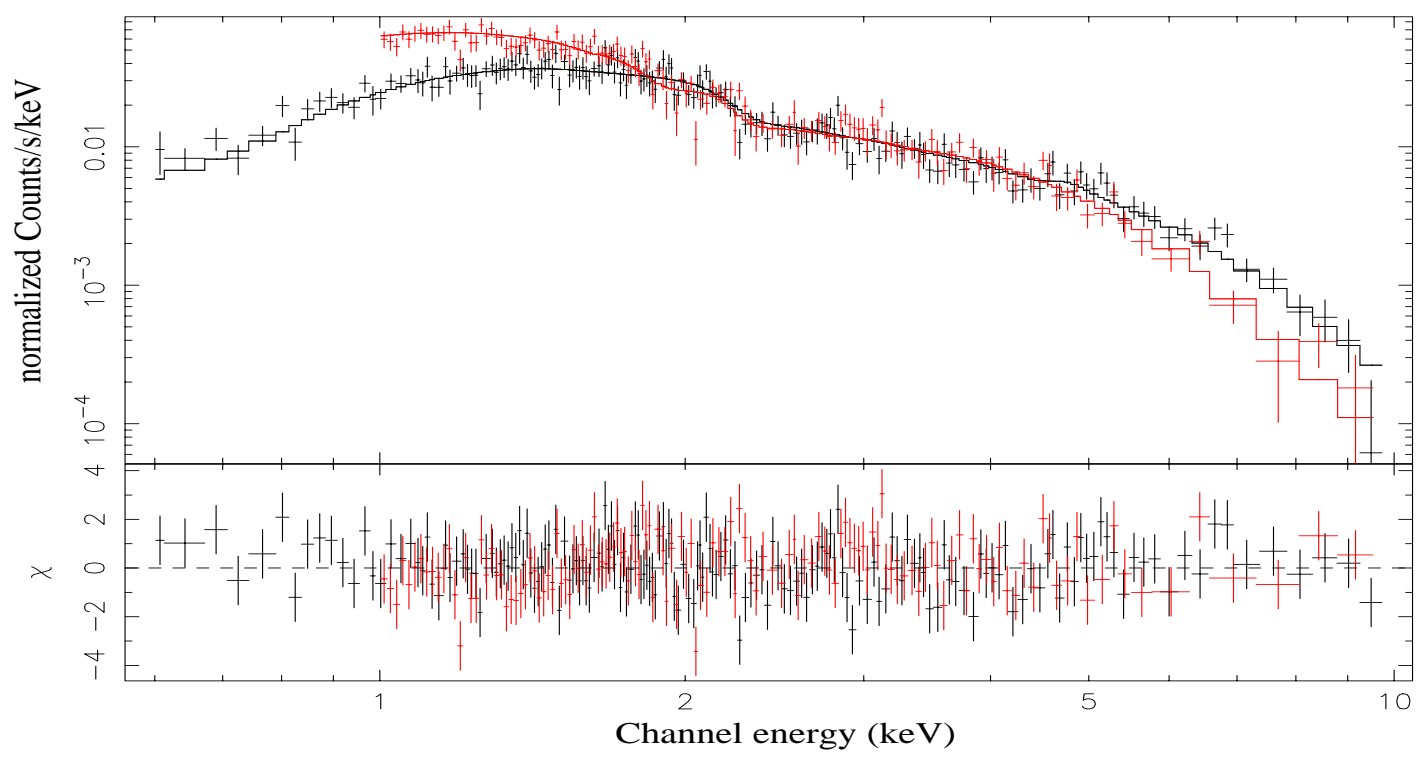

Fig. 4. The ASCA SIS01 and GIS23 folded spectra of NGC 7679 fitted with a power law model (see Table 3). The residuals of the fit are also shown.

The X-ray spectrum does not show any evidence of the characteristic cut-off at low energies and of the strong Iron-K line(s) at $\sim 6.4-6.9 \mathrm{keV}$ usually found in absorbed type 2 Seyferts with $N_{\mathrm{H}} \sim 10^{23}-10^{24} \mathrm{~cm}^{-2}$ (see Bassani et al. 1999 and references therein). Thus a Compton thin Seyfert 2 could be excluded.

One interesting possibility could be a Compton thick $\left(N_{\mathrm{H}} \gtrsim 5 \times 10^{24} \mathrm{~cm}^{-2}\right)$ Seyfert 2 galaxy. In this case the observed X-ray spectrum should be totally dominated by scattered radiation from an otherwise invisible nucleus. Typical examples of this class of "Compton Thick" sources are NGC 6240 (Vignati et al. 1999) and NGC 4945 (Guainazzi et al. 2000a). However the observed X-ray properties suggest against this hypothesis. First, we do not see the very strong ( $E W \sim 1-2 \mathrm{keV}$ ) Iron-K line usually observed in "reflection dominated" Seyfert 2 (Matt et al. 2000; Bassani et al. 1999). Second, as pointed out by Guainazzi et al. (2000b) in the case of IRAS $12393+3520$, the observed variability introduces severe constraints on this possibility since for $t_{\mathrm{var}} \sim 15 \mathrm{ksec}$ the scattering/absorption should occur very close to the nucleus ( $\sim$ a few $\times 10^{-4} \mathrm{pc}$ ), in net contrast with the idea that the matter obscuring the nuclear region is located at a distance of 1-100 pc. Finally, the good agreement between the LECS/MECS spectra with the PDS data up to $50 \mathrm{keV}$ excludes the presence of any absorption cutoff which is expected around $20 \mathrm{keV}$ for Compton-thick objects.

To conclude, the only kind of AGN consistent with the X-ray properties of NGC 7679 is a Seyfert 1 AGN. However, its broad-band properties make NGC 7679 a peculiar Seyfert 1 galaxy. First of all, the detection of a broad $\mathrm{H} \alpha$ component without a broad $\mathrm{H} \beta$ component is more typical of type 1.9 Seyferts, which are usually characterized by X-ray absorbed spectra (see Bassani et al. 1999 and references therein). Second, NGC 7679 is underluminous in the optical emission lines with respect to "normal" Seyfert 1. Using the $L_{(2-10 \mathrm{keV})}-L_{\mathrm{H} \alpha}$ relationship observed for Seyfert 1's and QSO (see Maiolino et al. 2001) and given the observed $L_{(2-10 \mathrm{keV})} \sim 8 \times 10^{42} \mathrm{ergs} \mathrm{s}^{-1}$, we would expect an $\mathrm{H} \alpha$ luminosity in the range $(1 \sigma) \sim 4 \times$ $10^{41} \sim 6 \times 10^{42} \mathrm{ergs} \mathrm{s}^{-1}$. This is at least a factor of 5 higher 
than the observed broad $\mathrm{H} \alpha$ luminosity $\left(\sim 8 \times 10^{40} \mathrm{erg} \mathrm{s}^{-1}\right.$, Keweley, private communication) in NGC 7679.

\subsection{Implications}

If we combine the results presented here (i.e. clear predominance of an AGN in the X-ray regime) together with the clear predominance of a starburst in the optical and IR regime (see Sect. 2) we conclude that we have discovered another "Composite" galaxy.

Although Starburst/Seyfert composites have been studied in the last two decades, we find here that the broad-band properties of NGC 7679 are different from most of the composite galaxies studied so far. Composite Starburst/Seyfert galaxies were first discovered and discussed by Veron et al. (1981a) and Veron et al. (1981b); similar objects were also found in the EMSS (Halpern et al. 1995), among serendipitously detected IR-bright Einstein sources (Moran et al. 1996a) and in deep ROSAT fields (Boyle et al. 1995; Griffiths et al. 1996). Composite Seyfert 2/Starburst galaxies have been recently studied by Levenson et al. (2001) and the AGN/starburst inter-connection has been also used to explain the overall spectral properties of the ULIRG (Iwasawa 1999). However, in all these objects it is now clear that heavy absorption in the nuclear region and a strong circumnuclear starburst are responsible for their broad-band properties (e.g. infrared, optical and X-ray regime, see e.g. Iwasawa 1999; Levenson et al. 2001). In particular, much of the observed far-infrared emission may be associated with the starburst, which also can contribute significantly to the observed X-ray flux in the soft $(E<$ few keV) energy band; the nuclear X-ray source is generally absorbed with $N_{\mathrm{H}}>10^{23} \mathrm{~cm}^{-2}$. In these objects the circumnuclear starburst should also play a significant role in the obscuration process (Levenson et al. 2001; Levenson et al. 2001). The most striking peculiarity of NGC 7679, at odds with the above examples, is that its $X$-ray spectrum is not highly absorbed $\left(N_{\mathrm{H}}<4 \times 10^{20} \mathrm{~cm}^{-2}\right)$ and strong $(E W \sim 1 \mathrm{keV})$ Iron lines (which could suggest a Compton-thick type 2 AGN) are not present.

Have other galaxies with X-ray, optical and infrared properties similar to NGC 7679 been detected before? Six ${ }^{5}$ low redshift galaxies, with optical spectra dominated by the features of HII galaxies and by the presence of weak and elusive $\mathrm{H} \alpha$ broad wings (probably indicative of a faint or obscured AGN) but X-ray luminosities typical of broad line $\mathrm{AGNs}_{\mathrm{S}}$ (i.e. $L_{x}$ ranging from $1.5 \times 10^{42} \mathrm{erg} \mathrm{s}^{-1}$ to $5 \times 10^{43} \mathrm{erg} \mathrm{s}^{-1}$ in the ROSAT band) have been singled out in a large spectroscopic optical survey of 210 bright IRAS and X-ray selected sources (Moran et al. 1996b). The $L(0.1-2.4 \mathrm{keV}) / L_{\mathrm{FIR}}$ and the $\frac{\mathrm{O}[\mathrm{III}]}{\mathrm{H} \beta}$ flux ratio of

\footnotetext{
${ }^{5}$ One of the objects in Moran's list (IRAS 10113+1736) is no longer a valid candidate since subsequent observations of all the 7 objects in the original sample have shown that, for this object, the IR and the X-ray emission originate from different sources (Condon et al. 1998).
}

NGC 7679 are in agreement with the values shown by these starburst/Seyfert composites. Only one of the objects in the Moran et al. (1996b) list has been studied in detail (IRAS 00317-2142; Georgantopoulos 2000) using ASCA data, while for the remaining 5 objects only low signal-to-noise ROSAT All Sky Survey data are available at present and no spectral or variability X-ray properties are known to date. IRAS 00317-2142 has clear Seyfert 1 properties in the X-ray domain; in particular, it has a low absorption column density and no other signs of possible obscuration (e.g. strong Iron lines). It is therefore very similar to NGC 7679 in many aspects. Another object with overall properties very similar to NGC 7679 (but not included in Moran's list) could be IRAS 12393+3520, which has been investigated in detail by Guainazzi et al. (2000b) using PSPC and ASCA data. The interpretation for these objects is that the optical spectra are influenced heavily by the starburst component, while the Seyfert component is responsible for most of the intense X-ray emission. As pointed out by Moran et al. (1996b), their peculiarity is not the strength of their starburst but the apparent optical weakness of the AGN. It is possible that a dusty ionized absorber is able to selectively obscure the optical emission, leaving the X-ray emission almost unabsorbed (Georgantopoulos 2000; Maiolino et al. 2001). However, the physical nature of these objects, the reconciliation of their optical and X-ray spectra, their role inside the Unification Scheme of AGN and the understanding of how the absorption process works in these objects and modifies their global properties are far from obvious and are still unknown.

\section{Conclusions}

On the basis of the spectral shape, the high luminosity, and the observed X-ray variability, we conclude that the X-ray emission of NGC 7679 is strongly dominated by the presence of an AGN. The starburst activity, revealed by the IR emission, by optical spectroscopy as well as by $\mathrm{H} \alpha$ imaging, and dominating the optical and IR bands, is clearly overwhelmed by the AGN in the X-ray band. At first glance, this is similar to what is observed in other composite starburst/AGN galaxies, e.g. NGC 6240 (Vignati et al. 1999) and NGC 4945 (Guainazzi et al. 2000b). However, at odds with the above examples, the X-ray spectrum of NGC 7679 does not show any signs of absorption.

What are the most important implications of this result? Objects like NGC 7679 show that the absorption process can work under very different scenarios. In this object we have unabsorbed X-ray emission but possible absorbed (or intrinsically weak) optical emission from an AGN. This scenario must be understood and included in the general framework of the AGN Unification Scheme, along with the usual type 1 and type 2 AGN, the Broad absorption line QSOs etc. (see Gilli et al. 2001 for an observational and updated summary on how the absorption process modifies the optical and X-ray properties in the AGN family). 
From an observational point of view, optical and infrared spectroscopy could be highly inefficient in revealing the presence of an AGN in objects like NGC 7679, which is unambiguously revealed by X-ray spectroscopic and variability investigations. This consideration has strong implications for the optical identification work of the faint and low $S / N$ X-ray sources in the XMM and Chandra fields; in this case the X-ray data are usually not good enough to perform a detailed X-ray investigation and the optical and infrared observations could misidentify the real source of the X-ray emission. Finally, the observed properties of NGC 7679 (e.g. the existence of a bar, the signs of tidal distortion with a nearby faint companion, etc. see Sect. 2) could make it a low-redshift example of the peculiar and interacting galaxies found by deep HST-NICMOS observations. Their number density seems to increase with $z$, being $\sim 4 \%$ of all galaxies locally and $\sim 14 \%$ at $z \sim 1$ (see Corbin et al. 2000 and references therein). We believe that the new generation of X-ray satellites, like XMM-Newton and Chandra, will find many sources similar to NGC 7679 and will help us investigate their nature in detail.

Indeed galaxies with bright X-ray emission $\left(L_{x} \sim\right.$ $10^{42-43} \mathrm{ergs} \mathrm{s}^{-1}$ ) but weak or absent AGN features in the optical band have been known since Einstein observations (Elvis et al. 1981; Tananbaum et al. 1997) and are now showing up in Chandra deep-fields (e.g. Hornschemeier et al. 2001; Fiore et al. 2000). Although these sources usually lack evidence for strong starburst emission and, therefore, their nuclei are not usually selected for more detailed and deeper optical studies, the main problem is the same as for objects like NGC 7679, i.e. to explain the optical weakness/disappearance of the AGN in these $\mathrm{X}$-ray luminous sources.

Acknowledgements. RDC wish to thank ESO in Garching for the hospitality during the preparation of part of this work. We thank A. Guarnieri and V. Zitelli for the optical spectroscopic observation of NGC 7679 at the Loiano Telescope, L. Kewley for having given us many results on NGC 7679 in advance of publication and A. Caccianiga, A. Comastri and P. Severgnini for useful discussions. This research has made use of SAXDAS linearized and cleaned event files produced at the BeppoSAX Science Data Center, the ASCA archive, the TARTARUS database. and the NASA/IPAC extragalactic database (NED). We thank all the members working at the above mentioned projects. This work received partial financial support from ASI (ARS-9974) and MURST (Cofin00-02-36).

\section{References}

Bassani, L., Dadina, M., Maiolino, R., et al. 1999, ApJS, 121, 473

Boyle, B. J., McMahon, R. G., Wilkes, B. J., \& Elvis, M. 1995, MNRAS, 276, 315

Boella, G., Chiappetti, L., Conti, G., et al. 1997, A\&AS, 122, 327

Cappi, M., Persic, M., Bassani, L., et al. 1999, A\&A, 350, 777
Chiappetti, L., \& dal Fiume, D. 1997, Data Analysis in Astronomy V, 101

Condon, J. J., Yin, Q. F., Thuan, T. X., \& Boller, T. 1998, AJ, 116, 2682

Combes, F. 2000, Lectures given at GH Advanced Lectures on the Starburst-AGN Connection, INAOE, June 2000, ed. D. Kunth, \& I. Aretxaga [astro-ph/0010570]

Corbin, M. R., Vacca, W. D., O'Neil, E., et al. 2000, AJ, 119, 1062

Dahlem, M., Weaver, K. A., \& Heckman, T. M. 1998, ApJS, 118,401

Dahari, O. 1985, ApJS, 57, 643

Della Ceca, R., Griffiths, R. E., \& Heckman, T. M. 1997, ApJ, 485,581

Durret, F., \& Warin, F. 1990, A\&A, 238, 15

de Vaucouleurs, G., de Vaucouleurs, A., Corwin, Jr. H. G., et al. 1991, Third Reference Catalogue of Bright Galaxies (Springer Verlag, New York)

Elvis, M., Schreier, E. J., Tonry, J., Davis, M., \& Huchra, J. P. 1981, ApJ, 246, 20

Fabbiano, G., Kim, D., \& Trinchieri, G. 1992, ApJS, 80, 531

Fabian, A. C., Barcons, X., Almaini, O., \& Iwasawa, K. 1998, MNRAS, 297, L11

Fiore, F., Guainazzi, M., \& Grandi, P. 1999, Handbook for NFI spectral analysis (http://www.tesre.bo.cnr.it/Sax/software/)

Fiore, F., et al. 2000, New Astron., 5, 143

Frontera, F., Costa, E., dal Fiume, D., et al. 1997, A\&AS, 122, 357

Georgantopoulos, I. 2000, MNRAS, 315, 77

Gilli, R., Risaliti, G., Severgnini, P., et al. 2001, ASP Conf. Ser. [astro-ph/0103077]

Griffiths, R. E., Della Ceca, R., Georgantopoulos, I., et al. 1996, MNRAS, 281, 71

Griffiths, R. E., Ptak, A., Feigelson, E. D., et al. 2000, Science, 290,1325

Guainazzi, M., \& Matteuzzi, G. 1997, SDC-TR-014, available at ftp:www.sdc.asi.it/

Guainazzi, M., Matt, G., Brandt, W. N., et al. 2000a, A\&A, 356,463

Guainazzi, M., Dennefeld, M., Piro, L., et al. 2000b, A\&A, 355, 113

Hagen, H.-J., Engels, D., \& Reimers, D. 1997, A\&A, 324, L29

Halpern, J. P., Helfand, D. J., \& Moran, E. C. 1995, ApJ, 453, 611

Hartmann, D., \& Burton, W. B. 1997, Atlas of Galactic Neutral Hydrogen (Cambridge; New York: Cambridge University Press)

Heckman, T. M. 2000, In Gas \& Galaxy Evolution Conference Proceedings [astro-ph/0009075]

Ho, L. C., Filippenko, A. V., \& Sargent, W. L. W. 1997, ApJS, 112,315

Hornschemeier, A. E., et al. 2001, ApJ, in press [astro-ph/0101494]

Kewley, L. J., Heisler, C. A., \& Dopita, M. A. 2001, ApJS, 132, 37

Iwasawa, K. 1999, MNRAS, 302, 96

Levenson, N. A., Weaver, K. A., \& Heckman, T. M. 2001, ApJ, 550,230

Levenson, N. A., Cid Fernandes, R., Weaver, K. A., et al. 2001, ApJ, in press

Maiolino, R., Marconi, A., \& Oliva, E. 2001, A\&A, 365, 37

Manzo, G., Giarrusso, S., Santangelo, A., et al. 1997, A\&AS, 122,341 
Matt, G., Fabian, A. C., Guainazzi, M., et al., MNRAS, 318, 173

Moran, E. C., Helfand, D. J., Becker, R. H., \& White, R. L. 1996a, ApJ, 461, 127

Moran, E. C., Halpern, J. P., \& Helfand, D. J. 1996b, ApJS, 106,341

Moran, E. C., Lehnert, M. D., \& Helfand, D. J. 1999, ApJ, 526,649

Nandra, K., George, I. M., Mushotzky, R. F., et al. 1997, ApJ, 477,602

Parmar, A. N., Martin, D. D. E., Bavdaz, M., et al. 1997, A\&AS, 122, 309

Pietsch, W., Roberts T. P., Sako, M., et al. 2001, A\&A, 365, L174

Pogge, R. W., \& Eskridge, P. B. 1993, AJ, 106, 1405

Ptak, A., Serlemitsos, P., Yaqoob, T., \& Mushotzky, R. 1999, ApJS, 120, 179
Tanaka, Y., Inoue, H., \& Holt, S. S. 1994, PASJ, 46, L37

Tananbaum, H., Tucker, W., Prestwich, A., \& Remillard, R. 1997, ApJ, 476, 83

Terashima, Y., Ho, L. C., Ptak, A. F., et al. 2000, ApJ, 533, 729

Terlevich, R., Tenorio-Tagle, G., Franco, J., \& Melnick, J. 1992, MNRAS, 255, 713

Veilleux, S., \& Osterbrock, D. E. 1987, ApJS, 63, 295

Veilleux, S. 2000, Proceedings of the Ringberg Meeting Starbursts - Near and Far [astro-ph/0012121]

Veron, P., Veron, M. P., Bergeron, J., \& Zuidervijk, E. J. 1981a, A\&A, 97, 71

Veron, P., Veron, M. P., \& Zuiderwijk, E. J. 1981b, A\&A, 102, 116

Vignati, P., Molendi, S., Matt, G., et al. 1999, A\&A, 349, L57

Weaver, K. A., Gelbord, J., \& Yaqoob, T. 2001, ApJ, 550, 261 